\title{
Introduction aux articles sur la morphologie des spermatozoïdes
}

\author{
Roger MIEUSSET
}

Rédacteur en chef

Le projet de publier dans Andrologie un ensemble d'articles sur le thème de la morphologie des spermatozoïdes est né lors du comité de rédaction de décembre 2005 sur la proposition de Martine Albert, Marie Roberte Guichaoua et Valérie Mitchell. La gestation fut assidue, et la parution de ces articles dans le présent numéro fut délibérément choisie pour faire suite à la session 'Morphologie des spermatozoïdes et fertilité' du congrès de la SALF à Colmar en décembre 2007, dont la richesse de la discussion est l'un des reflets non seulement du franc succès de cette session, mais aussi des difficultés soulevées par l'interprétation et l'intérêt en pratique de ce paramètre de la qualité des spermatozoïdes.

Aborder la question de la forme des spermatozoïdes en 2008 peut paraître quelque peu passéiste. En effet, la morphologie des spermatozoïdes reste le paramètre suscitant le plus de contradictions dans la littérature, que ce soit comme critère d'appréciation de la qualité des spermatozoïdes dans le bilan d'une infécondité masculine, ou comme élément prédictif du pouvoir fécondant des spermatozoïdes.

L'analyse des spermatozoïdes en microscopie optique, effectuée depuis un demi siècle, a donné naissance à de multiples classifications dont trois sont utilisées comme référence, la classification de Kruger-Tyberg, la classification de l'OMS, et celle de David, redéfinie par Auger et Eustache dans le souci d'une démarche d'assurance de qualité en biologie de la reproduction.

Dans un premier article, Marie Roberte Guichaoua et al. traitent de la morphologie des spermatozoïdes, observée en microscopie optique, dans les populations d'hommes fertiles et dans ses relations à l'infécondité et à la fécondation in vitro, discutent les rapports entre anomalie de la tête des spermatozoïdes et anomalie du contenu chromosomique, puis abordent les relations entre morphologie des spermatozoïdes et apoptose. Si les tératozoospermies polymorphes sont les plus fréquentes, les tératozoospermies monomorphes constituent des syndromes pour certains bien définis dont la présence oriente la conduite à tenir en termes de technique d'AMP et de suivi, comme cela est illustré dans un article compagnon avec le syndrome des spermatozoïdes macrocéphales.

Cependant, l'approche morphologique des spermatozoïdes en microscopie optique s'est enrichie en 2001 d'une nouvelle méthode d'observation à un fort grossissement pouvant aller jusqu'à plus de $\times 10$ 000, le MSOME (pour Motile Sperm Organelle Morphology Examination), qui a depuis été utilisée dans plusieurs centre d'AMP pour sélectionner le spermatozoïde à injecter en ICSI, prenant alors le nom d'IMSI (pour Intracytoplasmic Morphologically Selected sperm Injection).
Martine Albert et al., dans un deuxième article, développent la méthodologie de cette étude de la morphologie des spermatozoïdes et ses contraintes, décrivent l'observation de ces cellules à fort grossissement, abordent la classification des anomalies retrouvées à partir de leur propre expérience ainsi que l'intérêt du MSOME dans le cadre de l'IMSI, puis tracent les perspectives de cette nouvelle méthodologie d'évaluation de la forme des spermatozoïdes en soulevant d'importantes questions à propos de la codification rigoureuse de l'analyse du spermatozoïde sélectionné pour être injecté dans l'ovocyte, de la relation structure-fonction du spermatozoïde, et de l'intérêt du MSOME au plan diagnostique.

Mais il est des situations où l'analyse morphologique des spermatozoïdes en microscopie optique, quel que soit le grossissement utilisé, est insuffisante. Seule l'observation en microscopie électronique permet d'étudier les organites de cette cellule et de quantifier les anomalies des constituants impliqués dans l'aptitude migratoire et la fécondance du spermatozoïde. Valérie Mitchell et al., en collaboration avec Denise Escalier, traitent dans un troisième article des altérations des spermatozoïdes en microscopie électronique. Elles posent les indications de cette analyse, rappellent la structure et l'ultrastructure du spermatozoïde normal, décrivent les principaux phénotypes pathologiques de la tête du spermatozoïde et de l'attachement tête-flagelle, puis les principaux phénotypes flagellaires avec leur prévalence, leurs aspects moléculaires et leurs composantes génétiques. Elles abordent aussi les relations entre phénotypes pathologiques, aneuploïdie et anomalies de la structure de la chromatine, et passent en revue le potentiel de fertilité des phénotypes pathologiques et leur pronostic en AMP.

L'ensemble de ces articles, à la fois très documentés au plan scientifique et didactiques dans leur composition, servira, à n'en pas douter, à éclairer les lecteurs peu familiarisés avec l'analyse morphologique des spermatozoïdes en microscopie optique et électronique. Les données analysées pourront constituer un outil appréciable tant au clinicien, qui trop souvent encore ne sait que faire de toutes ces anomalies des spermatozoïdes, qu'au biologiste de l'AMP, pour qui parfois encore la présence d'au moins un spermatozoïde est suffisante à poser l'indication d'ICSI... Bref, l'étude de la morphologie des spermatozoïdes, en microscopie optique ou électronique, n'est pas qu'une fastidieuse technique de laboratoire consommatrice de temps se soldant par une multitude de détails dépourvus de sens pour le profane, mais un outil d'analyse d'une richesse non encore pleinement mesurée dans le diagnostic, l'exploration et le traitement de l'infécondité masculine. 